\title{
Christoph Asendorf, Super Constellation. L'influence de l'aéronautique sur les arts et la culture
}

Paris, Éditions Macula, 2013

\section{Nathalie Roseau}

\section{OpenEdition \\ Journals}

Édition électronique

URL : http://journals.openedition.org/artefact/736

DOI : 10.4000/artefact.736

ISSN : 2606-9245

Éditeur :

Association Artefact. Techniques histoire et sciences humaines, Presses universitaires du Midi

\section{Édition imprimée}

Date de publication : 15 juin 2017

Pagination : 225-229

ISBN : 978-2-7535-6525-8

ISSN : 2273-0753

\section{Référence électronique}

Nathalie Roseau, «Christoph Asendorf, Super Constellation. L'influence de l'aéronautique sur les arts et la culture », Artefact [En ligne], 5 | 2016, mis en ligne le 15 novembre 2017, consulté le 24 septembre 2020. URL : http://journals.openedition.org/artefact/736 ; DOI : https://doi.org/10.4000/artefact.736

Ce document a été généré automatiquement le 24 septembre 2020.

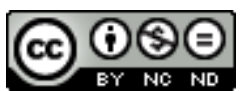

Artefact, Techniques, histoire et sciences humaines est mise à disposition selon les termes de la Licence Creative Commons Attribution - Pas d'Utilisation Commerciale - Pas de Modification 4.0 International. 


\section{Christoph Asendorf, Super Constellation. L'influence de l'aéronautique sur les arts et la culture}

Paris, Éditions Macula, 2013

Nathalie Roseau

\section{RÉFÉRENCE}

Christoph Asendorf, Super Constellation. L'influence de l'aéronautique sur les arts et la culture, avant-propos par Angela Lampe, traduit de l'allemand par Didier Renault et Augustine Terence, Paris, Éditions Macula, 2013, 528 p.

1 Publié en 1997 en allemand, Super Constellation, le livre de Christoph Asendorf, est enfin traduit en français grâce aux éditions Macula qui ajoutent à leur catalogue cet ouvrage monumental, ainsi qu'à Angela Lampe, commissaire de l'exposition Vues d'en haut, montée en 2013 au Centre Pompidou-Metz, qui en signe l'avant-propos. Sa publication intervient dans un contexte prolifique marqué en France par la sortie de plusieurs ouvrages d'histoire culturelle qui renouvellent l'historiographie aéronautique. Il faudra sans doute encore patienter avant que l'ouvrage ne franchisse l'Atlantique, sa navigation s'avérant plus lente que l'avion dont il porte le nom, le Super Constellation, premier avion de ligne à avoir traversé l'Atlantique en 1951, sans escale de New York à Paris, à une altitude de 7000 mètres. Sous cette bannière mythique, Asendorf choisit, à la manière d'un vol cosmique, de nous embarquer dans une ascension vertigineuse pour nous raconter l'invention d'un nouveau monde, notre monde.

Embrassant le $\mathrm{xx}^{\mathrm{e}}$ siècle, l'ouvrage saisit d'abord par son ambition encyclopédique. Des mathématiques à l'architecture, de la sculpture à la photographie, de la physique à la psychologie perceptive, du cinéma à l'urbanisme, la dimension chorale du livre 
exemplifie l'écriture panoramique du livre, survolant l'espace-temps, traçant des trajectoires qui construisent l'aéronautique comme une force puissante perfusant les sociétés modernes. Car c'est bien là l'hypothèse de l'auteur, qu'il énonce dès l'ouverture du livre, se référant à l'historien d'art Fritz Wichert qui, dès 1909, suit l'intuition que "la conscience liée aux représentations visuelles subit des transformations dues à l'évolution de la technique " et examine les effets de la possibilité de voler (p. 22). Asendorf tisse une constellation de sources qui matérialise peu à peu un réseau dense de lieux et de liens. Des étoiles brillent pour nous guider dans ce cosmos: Robert Delaunay, László Moholy-Nagy, Alexandre Rodchenko, Reyner Banham, Thomas Pynchon, Stanley Kubrick. D'autres comme Carl Schmitt, considéré comme le juriste officiel du $\mathrm{III}^{\mathrm{e}}$ Reich, sont plus sombres mais essentielles pour comprendre les interférences (plus que les influences) et les moments d'apogée. L'écriture flotte et navigue, renforçant cette impression océanique, conférant au texte une poésie d'évocation sans jamais nous laisser orphelin de l'argumentation. Inauguré en 1909, le récit se compose de quatre parties qui suivent un fil diachronique, épousant l'histoire militaire, artistique, sociale, géopolitique. Il se double d'un panorama visuel, composé de cahiers à part qui offrent au lecteur la possibilité de se consacrer entièrement à l'image, inaugurant l'ouvrage avec la photographie du premier vol, en décembre 1903, des frères Wright en Caroline du Nord, le clôturant par celle de la médiathèque de Sendai, réalisée par Toyo Ito, dont nous comprenons à la fin la clarté limpide du choix. Comme une toile dont il s'agirait de saisir tous les détails, le lecteur finit dans une élévation, par l'embrasser pleinement comme une évidence.

3 «L'art est l'instrument d'une interprétation en profondeur des époques » (p. 406). Citant l'historien d'art Hans Seldmayr, Asendorf énonce son programme. À partir d'œuvres choisies, il décrit les liens du monde qui se tissent, relisant la société à travers l'objet aéronautique, décryptant celui-ci à travers les transformations de la science, de l'art et de la technique. Ce faisant, il déploie l'imaginaire des concepteurs comme le lieu de la coopération de mondes hétérogènes, entremêlant la fiction et la réalité, la seconde nourrissant la première qui la fait advenir. Le désir précède le réel comme nous le montre le beau texte du peintre Otto Piene, écrit antérieurement au premier vol spatial habité de Youri Gagarine: "Quand notre liberté sera-t-elle assez forte pour conquérir le ciel sans but, pour planer dans l'univers, y faire l'expérience du grand jeu de la lumière et de l'espace sans y être poussés par la peur et la méfiance ?» (p. 463).

4 Lorsqu'il paraît, l'avion promet tous les possibles et ouvre le champ du regard. Nadar avait déjà inauguré en 1858 la "pure sensation optique » de la vue aérienne qui montrait des aspects de la réalité jusqu'alors inaccessibles au regard. Visitant en 1912 le Salon de la locomotion aérienne à Paris, Marcel Duchamp contemple les objets bruts aéronefs, fuselages, hélices - et dit à Constantin Brancusi, qui l'accompagne avec Fernand Léger: "C'est fini la peinture! Qui fera mieux que cette hélice?» (p.49). Le Corbusier se passionne pour l'avion, non pas en tant que machine volante, mais comme construction légère. L'avant-garde technique touche au domaine perceptif, la statique étant remplacée par la dynamique, la vision aérienne « enchaînant les objets selon une apparence flottante» (p. 24). Le mathématicien Hermann Minkowsky annonce que «le schéma d'une conception quadridimensionnelle du continuum espace-temps a remplacé le temps et l'espace séparés» (p. 124). "La perfection de l'hélice n'apparaît que lorsqu'elle est en mouvement, nous dit quant à lui Karl Vollmöller. Dès qu'elle est en mouvement [...], elle n'est plus autre chose qu'une pure courbe mathématique» (p.49). La dynamique se double du panoramique, l'avion 
entraînant le dépassement du point de vue fixé, autorisant la mobilité du regard dans toutes les directions.

5 Saisis par le vertige de la technique, les artistes s'emparent du media aérien. Avec Équipe de Cardiff (1912-1913), Robert Delaunay condense le tableau de l'existence moderne, intégrant et dissociant les éléments mécaniques. "Les vues aériennes sont des condenseurs d'espace", écrit Alexandre Rodchenko dont les photographies s'émancipent de la représentation stable de l'espace (p. 81). L'abolition de la perspective devient une vertu révolutionnaire. L'émancipation des fondements affecte non seulement le sol, l'horizon, mais aussi de manière plus figurée les conceptions et les états de la société. Dans son article « la roue, l'hélice et la suite ", Lissitzky affirme : « l'homme volant est sur un seuil. [...] Il faut libérer une énergie nouvelle qui nous offre un mode de locomotion nouveau » (p. 118).

6 La technique devient "cape d'invisibilité » (p. 151). Dans son livre Von Material zu Architektur, Moholy-Nagy propose de dépasser «l'homme sectoriel ». Derrière cela, se dessine le concept de l'organique que la conception aéronautique va épouser avec le streamlining et l'aérodynamisme et que les artistes vont célébrer dans Aircraft ou l' Aeropittura. L'arc et la parabole incarnent les formes symboliques d'unicité et d'organicité. L'espace devient une entité illimitée que Henri Matisse développe dans ses trois versions de La Danse, donnant «avec une surface limitée, l'idée de l'immensité » (p.195). Le vertige de l'espace sans repères provoque la confusion des sens. Paul Valéry publie La conquête de l'ubiquité. Pour Robert Musil, auteur de L'homme sans qualités, l'indétermination est la marque de l'époque, tandis que la physique met au point la théorie de la relativité. Des voix pessimistes s'élèvent toutefois comme celles de l'historien d'art Aby Warburg pour qui les frères Wright sont les «destructeurs fatidiques de la notion de distance» (p.196). L'historien d'art Bernard Berenson entrevoit quant à lui la naissance d'un art qui anéantira la conception de son espace tandis que Gertrude Stein, voyant d'avion le sol comme une toile cubiste, comprend qu'« un créateur est toujours un contemporain. Avant tout le monde, il connaît ce que les autres ne savent pas encore » (p. 265).

7 L'avion est une machine à voir, littéralement et métaphoriquement, ce qui fera dire à Paul Virilio, dans une forme d'inversion, que «le cinéma, ce n'est pas je vois, c'est je vole»(p.75). Les procédés cinématographiques montrent à leur tour la voie, influençant les dispositifs de simulation du vol. Avec son Vitarama, suivi de son Cinérama, Fred Waller recherche une synthèse entre panorama et cinématographie. Abel Gance projette, en 1927, son Napoléon sur trois écrans disposés en arc de cercle. Tentatives qui aboutissent, à la fin des années 1930, aux dispositifs présentés lors de l'exposition de New York avec le Futurama de Norman Bel Geddes et la périsphère de Henry Dreyfuss.

Les années 1930 sont le temps d'une nouvelle ère, révolutionnaire et menaçante, qui atteint un point culminant pendant le conflit mondial avec une forme de guerre inédite. "La guerre aérienne est l'une des plus brutales agressions portées contre l'édifice de la civilisation que puisse permettre la technique. Les anciens concepts d'espace, de territoire et d'État y perdent leur signification» (p. 323). Avec une partie magistrale sur la guerre aérienne, Asendorf marque ici la rupture que constitue l'utilisation de l'avion comme arme de destruction massive. Entre 1937 (à Guernica) et 1945, de nombreuses villes sont anéanties par l'Area bombing. Plusieurs centaines de milliers d'êtres humains trouvent la mort. La menace qui vient du ciel expose le sol qui 
partout peut être pris pour cible des bombardements. L'art et la guerre s'allient autour des techniques de camouflage, dont le cubisme entend assumer la paternité. Commande avait déjà été passée à des artistes vers la fin de la Première Guerre mondiale pour camoufler les navires de guerre. En 1941, le maire de Chicago, souhaitant examiner les possibilités de camouflage pour sa ville, s'adresse à Moholy-Nagy qui délègue à Gyorgy Kepes le soin de dispenser des cours de peinture de camouflage dans sa School of design. L'architecture des bunkers du mur de l'Atlantique marque à son tour les concepteurs. Prises comme cibles, les villes elles-mêmes sont invitées à se repenser. La protection antiaérienne prône la dissolution des concentrations urbaines par dissémination. L'expérience de la guerre aérienne redonne une actualité à la Charte d'Athènes et à son projet d'une déconcentration par la séparation des fonctions ; elle conforte Frank Lloyd Wright qui milite pour une décentralisation urbaine, tandis que, en 1946, Hans Scharoun annoncera, déclaration glaciale, depuis Berlin que "la "dispersion mécanique" par les raids aériens donne finalement la possibilité de "modeler un paysage urbain" » (p. 343).

Camouflé, dispersé, le sol pare à la menace en attaquant. Une nouvelle ligne de front se met en place en Allemagne, du nom de son inventeur, Josef Kammhuber, qui utilise la lumière comme système de défense mobile et flexible, pour éblouir et cibler. La guerre aérienne a désormais lieu dans un espace virtuel dont les frontières éphémères ne sont formées que par des flux d'énergie. L'ubiquité de la menace devient un état permanent, avec les missiles et les têtes nucléaires. En 1943, s'ouvre au Moma l'exposition Airways to Peace qui fait le pari de mettre la technologie guerrière au service de la paix et de la communication. Après guerre, Buckminster Fuller met au point un nouveau système de projection cartographique, la Dymaxion map, tandis qu'en Allemagne, Carl Schmitt développe son point de vue sur l'histoire mondiale avec le concept de révolution spatiale. Nous assisterions à " la délocalisation absolue et partant, au pur caractère d'anéantissement de la guerre aérienne moderne» (p. 371). Son analyse se teinte de couleurs apocalyptiques : le déracinement, la privation de toute sécurité, ouvrent, selon lui, à la peur du désordre ouverte par la libération de l'espace qui ne peut être endiguée que par la dictature.

10 Possédant de loin le plus important potentiel militaire, industriel et économique l'industrie aéronautique américaine est devenue la première du monde avec deux millions de travailleurs -, c'est aux États-Unis que revient naturellement la tâche de définir et de mettre en œuvre un nouvel agencement du grand espace mondial après guerre. One World, le livre de Wendell Wilkie, paraît en 1943, vendu à deux millions d'exemplaires. "Peut-être dans une époque éloignée, les inventions en matière de transports et de communications rendront-elles le One World possible» (p.403): William Ogburn, auteur de The social effects of aviation (1946), annonce que l'aviation va transformer l'urbanisme. La démesure se manifeste dans les créations d'une échelle inédite. Le Moma consacre, en 1947, une exposition au thème du Large Scale Modern Painting. Jackson Pollock, Max Ernst et Mark Tobey y développent leurs techniques du All over. Convaincu de la nécessité d'une collaboration entre les artistes et les architectes, Lucio Fontana déclare que l'avion est «la première architecture de l'ère spatiale », tandis que « la fusée sera l'architecture de l'avenir » (p. 460). Eero Saarinen crée à Saint-Louis la Gateway Arch et conçoit le Terminal TWA de New York, dont l'espace n'a plus de limites. Kenzo Tange recouvre quant à lui la baie de Tokyo d'une structure en réseaux organisationnelle. Sa mégastructure territoriale, permettant à tout lieu d'être atteignable, est proche des utopies de colonisation de l'environnement 
à grande échelle comme le sont également les projections de Reyner Banham et d'Archigram, ou le dôme géodésique présenté en 1967 par Fuller à l'exposition de Montréal.

11 Un monde dual apparaît, partagé entre la continuité de l'ordre et du contrôle, et la discontinuité des changements brusques et de la désintégration. La fusée l'incarne et Thomas Pynchon (qui a travaillé chez Boeing comme assistant de construction) consacre son Arc-en-ciel de la gravité. Vivre le nouvel espace aérien, c'est éprouver l'apesanteur et l'accélération. Avec 2001, L'Odyssée de l'espace, Stanley Kubrick invente son propre code visuel. L'accélération de la chute s'accompagne d'une impression d'immobilité. La simulation qu'opère le film est si réelle que le cosmonaute Alexeï Leonov confie avoir eu « la même impression que s'il était retourné une deuxième fois dans l'espace » (p. 475). La fiction peut aller au-delà du réel, l'anticipant, le sublimant. La conquête spatiale signe aussi le commencement du déclin et l'assombrissement des perspectives. Le bombardier B52 est pour le documentariste Hartmut Bitomsky «le sommet de la puissance de la civilisation occidentale », dans laquelle s'entremêlent technique et pouvoir de destruction (p.492). La fin de l'espace comme horizon inatteignable cède la place à l'avènement de l'information. L'être humain a deux corps, le corps réel de sa présence matérielle et le corps fictif de sa présence aux flux, dont les contours sont déterminés par l'information dirigée vers lui ou qu'il capte. La médiathèque de Sendai réalisée par Toyo Ito incarne ces deux corps, entièrement revêtue d'une peau de verre qui fait d'elle "un phénomène visuel polymorphe de lumières multicolores » (p. 502).

12 L'histoire de l'aéronautique peut se lire comme « une évolution qui va de la simulation des mouvements aux mouvements réels, pour céder la place aux seuls flux des images et des informations » (p. 476). Immobilis in mobili, l'homme aérien a conquis l'ubiquité, marqué par la confusion des sens, le vertige du déséquilibre, le choc de la distance illimitée et comprimée dans l'espace-temps. La face sombre de cette accélération se lit dans la puissance destructrice de l'arme aérienne et le glissement du totalisant au totalitaire. Entre invention et anéantissement, le funambule marche sur un chemin de crête. Enracinée en plusieurs lieux, la culture aérienne s'est mondialisée, diffusant dans toutes les sphères de la société, inventant la globalité.

\section{AUTEURS}

\section{NATHALIE ROSEAU}

École nationale des Ponts et Chaussées - LATTS 